\title{
Human Scavenger Receptor B1 Is Involved in Recognition of Apoptotic Thymocytes by Thymic Nurse Cells
}

\author{
Hitomi Imachi, Koji Murao, Chiharu Hiramine, Yositaka Sayo, Makoto Sato, \\ Hitoshi Hosokawa, Toshihiko Ishida, Tatsuhiko Kodama, Oswald Quehenberger, \\ Daniel Steinberg, and Jiro Takahara
}

First Department of Internal Medicine (HI, KM, YS, MS, HH, TI, JT), and Department of Health Science (CH), Kagawa Medical University, Kagawa, Japan; Research Center for Advanced Science and Technology (TK), University of Tokyo, Tokyo, Japan; and Department of Medicine (OQ, DS), University of California, San Diego, California

\begin{abstract}
SUMMARY: Recognition and uptake of apoptotic cells by neighboring phagocytes is essential for the clearance of dying cells without accompanying inflammation or tissue damage. In the thymus, many apoptotic cells are generated in the process of negative selection, and both thymic macrophages (professional phagocytes) and nursing thymic epithelial cells (nursing TEC; nonprofessional phagocytes) recognize and ingest them. However the receptors responsible for this recognition and uptake have not been identified. In the present study, we have established a human nursing TEC line and examined the expression of several genes of the scavenger receptor family considered to be potential receptors for apoptotic cells. Human scavenger receptor-B1 (hSR-B1)/CLA-1, previously shown to recognize apoptotic cells, was strongly expressed in nursing TEC, whereas there was little or no expression of the other scavenger receptors tested: scavenger receptor class $A$, CD36, or CD68. Suppression of hSR-B1/CLA-1 expression using antisense oligonucleotides decreased the binding of apoptotic thymocytes to nursing TEC by more than $40 \%$. These results indicate that hSR-B1/CLA-1 may play a major role in the clearance of apoptotic cells in the thymus, mediating the recognition and ingestion of apoptotic thymocytes by nursing TEC. (Lab Invest 2000, 80:263-270).
\end{abstract}

\begin{abstract}
A poptosis is a critically important physiologic mechanism by which large numbers of unwanted cells are targeted for death and removal (Raff, 1992). Cells undergoing apoptosis are recognized and ingested by phagocytes, without the release of inflammatory mediators. The alternative form of cell death, necrosis, is uncontrolled and accompanied by the release of potentially toxic and immunogenic intracellular contents from the apoptotic cells into the surrounding tissue. Macrophages are the professional phagocytes that remove apoptotic cells, but other cell types also participate, including epithelial and tumor cells, which can take up neighboring cells that undergo apoptosis. In this way large tracts of cells can be removed in an all but invisible manner, ie, without initiating an inflammatory response.

In the thymus, most immature thymocytes die via apoptosis as a result of negative selection, the failure of positive selection, and presumably the failure of the $\mathrm{T}$ cell receptor gene rearrangement (Shortman et al,
\end{abstract}

Received November 17, 1999.

This work was supported by the Uehara Memorial Foundation of Japan. Address reprint requests to: Dr. H. Imachi, First Department of Internal Medicine, Kagawa Medical University, 1750-1 Ikenobe, Kita-gun, Mikicho, Kagawa 761-0793, Japan. Fax: 8187891 2147; E-mail: ibitomi@kms.ac.jp
1991). Until recently it was thought that apoptotic thymocytes were phagocytosed mainly by adjacent macrophages (Savill et al, 1993; Surh and Sprent, 1994; Wekerle et al, 1980). However there is now evidence that the thymic nurse cell (TNC) is also a site of thymocyte apoptosis (Aguilar et al, 1994; Hiramine et al, 1996). The TNC, a thymic lymphoepithelial cell complex, consists of one thymic epithelial cell (TEC) and many thymocytes enclosed by epithelial cytoplasmic processes. It has been considered to be a specialized site for proliferation, differentiation, and maturation of immature thymocytes (Wekerle et al, 1980) and, recently, for positive and negative selection (Hiramine et al, 1995). We have previously reported methods for establishing murine nursing TEC lines capable of binding and enveloping viable immature thymocytes to form TNC (Hiramine et al, 1990, 1995). These nursing TEC lines can induce apoptosis mainly in small $\mathrm{CD} 4^{+} \mathrm{CD}^{+}$thymocytes as well as proliferation/ differentiation of immature thymocytes (Hiramine et al, 1990, 1995). Recently we have demonstrated that nursing TEC in vivo exhibit phagocytic activity in the digestion and clearance of apoptotic thymocytes as shown by the presence of phagolysosomes containing acid phosphatase activity (Hiramine et al, 1996). However, nursing TEC receptors for apoptotic thymocytes have not been defined. 
The mechanisms by which apoptotic cells are recognized and removed have received extensive attention in the last few years, and a number of receptors that may be involved have been identified in vitro (Krieger, 1997; Pearson, 1996; Savill et al, 1993), but which of these are involved in TNC/nursing TEC uptake of thymocytes has not been explored. A recent report shows that the macrophage scavenger receptor class A (SR-A) plays a role in the clearance of apoptotic thymocytes by thymic macrophages (Platt et al, 1996). CD36 is firmly established as a macrophage receptor for apoptotic cells (Savill et al, 1993). We have shown that human scavenger receptor-B1 (hSR-B1)/CLA-1 can act as a receptor for apoptotic thymocytes in vitro (Murao et al, 1997). In the present study, we have evaluated the relative importance of these receptors in the recognition of apoptotic thymocytes by human nursing TEC.

\section{Results}

A human nursing TEC line was isolated and established using methods described previously for isolation of mouse nursing TEC lines (Hiramine et al, 1990, 1995). These nursing TEC expressed desmosomes and cytokeratin, which are specific for epithelial cells but were negative for Mac-1 (data not shown). AntiMac-1 antibody does not label TEC, whereas macrophages and $80 \%$ of interdigitating cells in the thymus are positive for Mac-1 (Nabarra and Papiernik, 1988). These results indicated that the nursing TEC were readily distinguishable from macrophages.

We first examined apoptotic cell binding to the nursing TEC by transmission electron microscopy. Mouse thymocytes previously incubated with dexamethasone and demonstrating DNA laddering and morphological changes typical of apoptotic cells, were cocultured with the TEC. As shown in Figure 1, these nursing TEC in culture retained the ability to bind and internalize apoptotic thymocytes. The internalized apoptotic thymocytes were then digested by lysosomal enzymes, leaving small vacuoles containing a small amount of nondigestable, electron-dense material as previously described (Hiramine et al, 1996). This is in contrast to phagocytosis of apoptotic cells by macrophages, which does not lead to any accumulation of residual undigested material.

We then assessed the levels of expression in nursing TEC of three known receptors for apoptotic cells: hSR-B1/CLA-1, SR-A, and CD36. As shown in Figure 2A, hSR-B1/CLA-1 protein and mRNA were strongly expressed. Also, the cytoplasmic membrane of the nursing TEC stained positively with anti-hSR-B1/ CLA-1 antibody (data not shown).

As shown in Figure 2B, SR-A protein was present in nursing TEC, but the level was low, much lower than that in PMA-treated THP-1 cells. RT-PCR showed only very faint bands for SR-Al and SR-All mRNAs (Fig. 2B, bottom). CD36 is expressed by a limited range of cell types, namely monocyte-macrophages, microvascular endothelium, platelets, and some erythroid cells (Greenwalt et al, 1992). It has been recog-

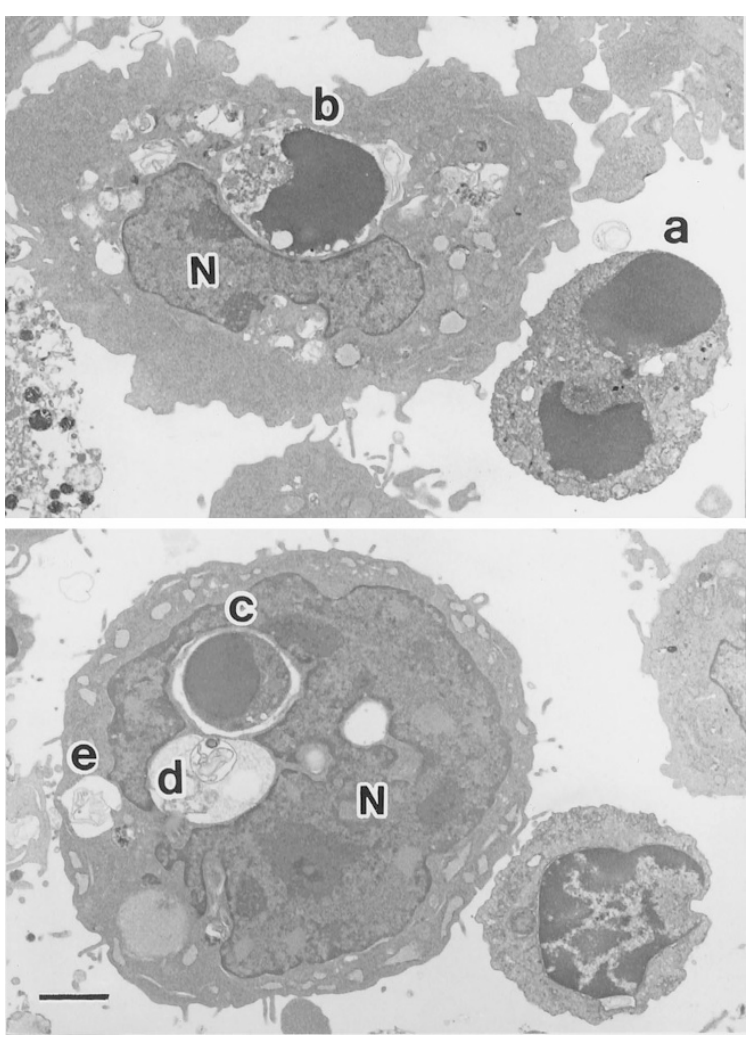

Figure 1.

Transmission electron micrograph of human nursing thymic epithelial cells (TEC) cocultured for 4 hours at $37^{\circ} \mathrm{C}$ with murine apoptotic thymocytes induced by dexamethasone. a, An apoptotic thymocyte with two nuclear fragments adhering to the surface of a nursing TEC. $b$, A partly degraded apoptotic thymocyte within a phagolysosome in a nursing TEC. $c$, An intact apoptotic body within a phagosome in a nursing TEC. $d$, An almost digested apoptotic body. e, A small vacuole with a small amount of nondigestable electron-dense material. $N$, Nucleus of a nursing TEC. Bar, $2 \mu \mathrm{m}$.

nized for some time as a receptor for apoptotic cells (Ren et al, 1995; Savill et al, 1993), and Ren et al (1995) recently reported that CD36 gene transfer can confer phagocytic capacity for cells undergoing apoptosis. As shown in Figure 2C (top), we were unable to detect surface expression of CD36 in nursing TEC using flow cytometric analysis even though we could readily detect CD36 surface expression in PMA-treated U937 cells, a human monocyte-derived cell line. Nor could we detect any CD36 mRNA by RT-PCR analysis (Fig. $2 \mathrm{C}$, bottom).

The next step was to use an inhibitor or antibody to SR-A or CD36 to determine their ability to inhibit binding of apoptotic cells by nursing TEC. Pretreatment with the SR-A ligand (Fadok et al, 1992a), fucoidan, did not inhibit the binding of apoptotic thymocytes to the nursing TEC (Fig. 3A). As expected, in the nursing TEC, anti-CD36 did not reduce the binding of apoptotic thymocytes, although this antibody inhibits the binding of apoptotic cells to human monocyte-derived macrophages (Fadok et al, 1998).

CD68, the human homolog of rodent macrosialin, has been implicated as a receptor for oxidized lowdensity lipoprotein (OxLDL) (Ramprasad et al, 1995). CD68 may, like other OxLDL receptors, recognize 
A

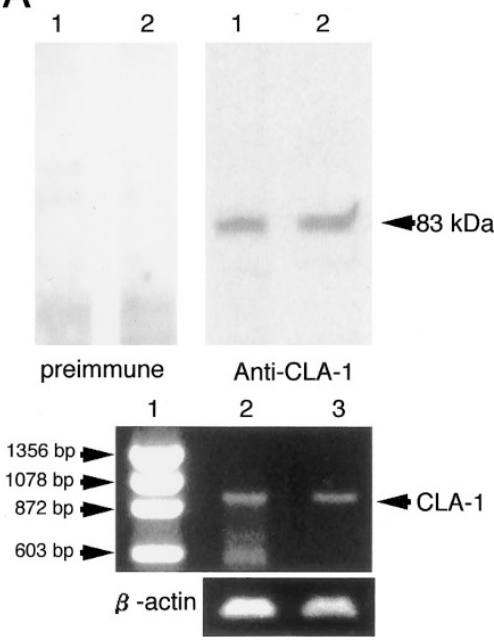

B

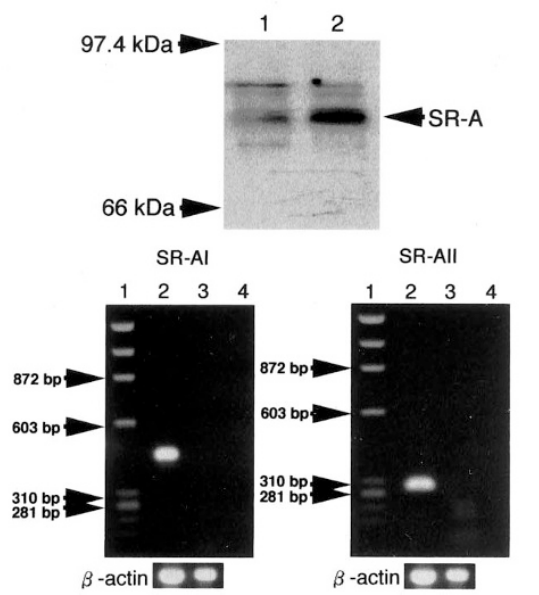

C
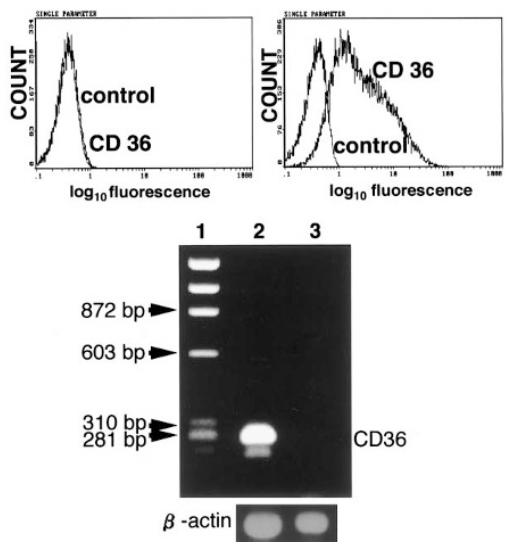

\section{Figure 2.}

Expression of scavenger receptors in human nursing TEC. A, Human scavenger receptor-B1 (hSR-B1)/CLA-1. Top: (Left) Preimmune serum as negative control; (right) immunoblot of hSR-B1/CLA-1 in cell lysates from human monocyte-derived THP-1 cells (lane 1) and nursing TEC (lane 2). Bottom: RT-PCR of hSR-B1/CLA-1 in nursing TEC. Lane 1, markers; lane 2, THP-1 cells; lane 3, nursing TEC. B, Scavenger receptor class A (SR-A). Top: Immunoblot of SR-A in THP-1 cells (lane 1) and in nursing TEC THP-1 cells (lane 2). Bottom: RT-PCR of SR-Al and SR-All, respectively. Lane 1, markers; lane 2, THP-1 cells; lane 3, nursing TEC; lane 4, control without reverse transcriptase. THP-1 cells were incubated with PMA (100 ng/ml) for 2 days. C, CD36. Top: Flow cytometric analysis for surface expression of CD36 in nursing TEC (left) and in human monocyte-derived cells U937 cells (right). Cells were labeled with control IgG (dotted line) or anti-CD36 (solid line). Bottom: RT-PCR analysis. Lane 1, markers; lane 2, U937 cells; lane 3, nursing TEC.

\section{A}

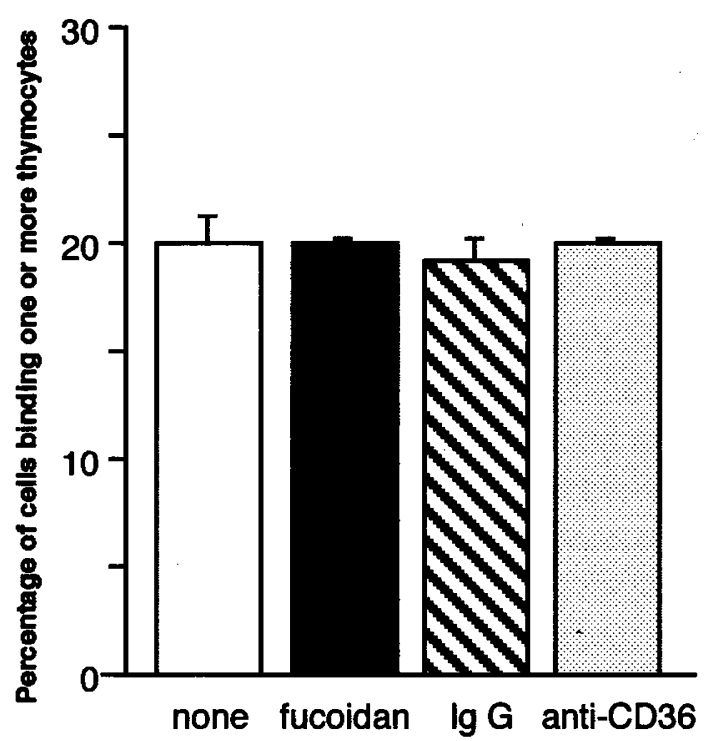

B

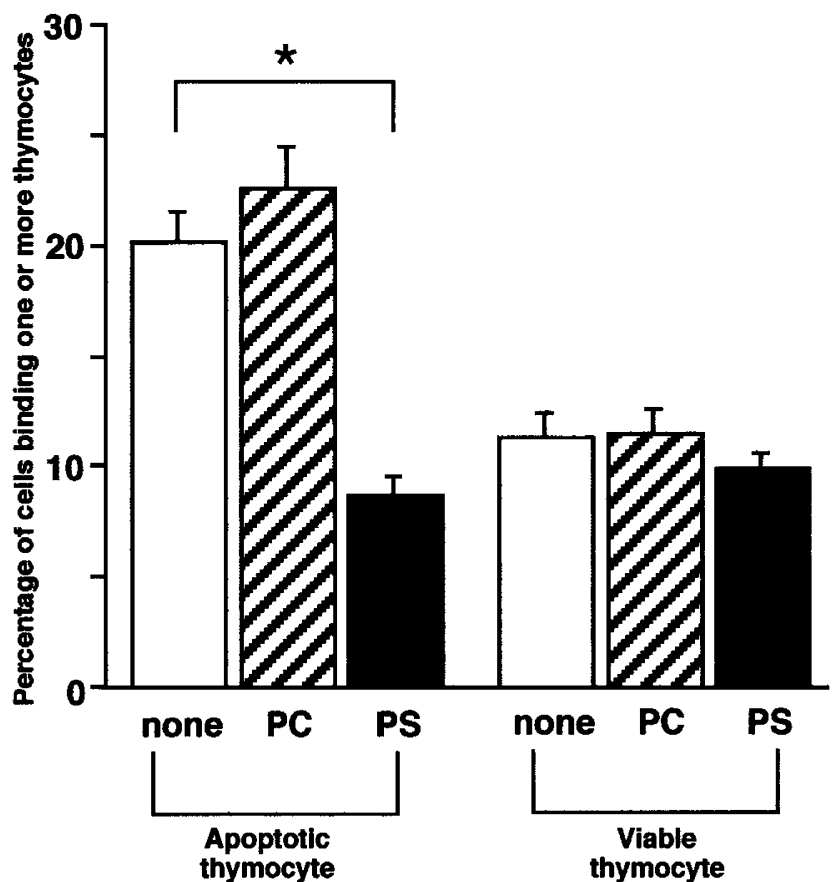

Figure 3.

Effects of various competitors on binding of apoptotic thymocytes to nursing TEC. A, Fucoidan was added at a final concentration of $100 \mu \mathrm{g} / \mathrm{ml}$. Nursing TEC were pretreated for 30 minutes with $100 \mu \mathrm{g} / \mathrm{ml}$ anti-CD36 and an isotype-matched control antibody before phagocytosing apoptotic thymocytes. Results are the mean \pm SE of three experiments. B, Phosphatidylserine (PS) liposomes, but not phosphatidylcholine (PC) liposomes, compete for apoptotic cell binding to nursing TEC. Binding of viable thymocytes was only about $50 \%$ that of apoptotic thymocytes and was not affected by PS liposomes. Final total lipid concentration was 0.1 mm. Results are the mean \pm SE of five experiments. *Significantly different $(p<0.005)$. 
apoptotic cells but was not detected in nursing TEC by FACS analysis (data not shown).

There is a considerable body of evidence showing that one mechanism by which macrophages recognize apoptotic cells is by recognition of excess phosphatidylserine (PS) on the plasma membrane (Fadok et al, 1992a, 1992b; Savill et al, 1993). Exposure of PS on the surface of apoptotic cells is associated with phagocytosis by macrophages of some phenotypes (Fadok et al, 1992a, 1993; Savill et al, 1990). Loss of membrane phospholipid asymmetry and early, external expression of PS have been documented with many different cell types undergoing apoptosis, and the mechanisms mediating this membrane change are under active investigation (Fadok et al, 1992a; Martin et al, 1995; Mower et al, 1994). Recent work by Ryeom et al (1996) shows that CD36 can recognize PS-rich liposomes under certain conditions even though it operates quite differently under other conditions (Savill et al, 1993). To determine whether recognition of PS plays a role in nursing TEC binding of apoptotic thymocytes, we tested for competition by PS-rich liposomes, as previously described (Murao et al, 1997). Figure 3B shows that PS liposomes, but not phosphatidylcholine (PC) liposomes, strongly inhibited the binding of apoptotic cells to the nursing TEC. The profile of binding was also changed. The percentage of nursing TEC binding two or more/one or more apoptotic thymocytes was $36.8 \pm 3.6 \%$ in PC treatment. In contrast, PS treatment reduced the percentage of nursing TEC binding two or more thymocytes to $11.6 \pm 2.3 \%$. There was some binding of untreated thymocytes also, but this was not affected by either PS or PC liposomes. These findings are in agreement with our previous observations on hSR-B1/CLA-1transfected cells (Murao et al, 1997), showing that binding of apoptotic thymocytes was almost completely prevented by unilamellar liposomes containing PS but not by liposomes containing PC.

Finally, to estimate the extent to which hSR-B1/ CLA-1 accounted for the recognition of apoptotic thymocytes by nursing TEC, antisense oligonucleotides were used. As shown in Figure 4, antisense oligonucleotides against hSR-B1/CLA-1 suppressed hSR-B1/CLA-1 protein expression in nursing TEC but had no effect on cells transfected with the sense oligonucleotide. In contrast, these oligonucleotide treatments had no effects on the steady-state expression of cyclophilin $A$ as negative control. The cells transfected with the antisense oligonucleotide showed a marked decrease in binding of apoptotic cells, to a level about $40 \%$ of that in the cells transfected with the sense oligonucleotide. Furthermore, the antisense oligonucleotide-treatment reduced the percentage of the nursing TEC binding multiple thymocytes to about one third compared with that in sense oligonucleotide treatment.

These results suggest that hSR-B1/CLA-1 plays a major role in the clearance of apoptotic thymocytes by nursing TEC in the thymus and that this is linked at least in part to its recognition of PS on the membrane of the target cells. The low levels of expression of

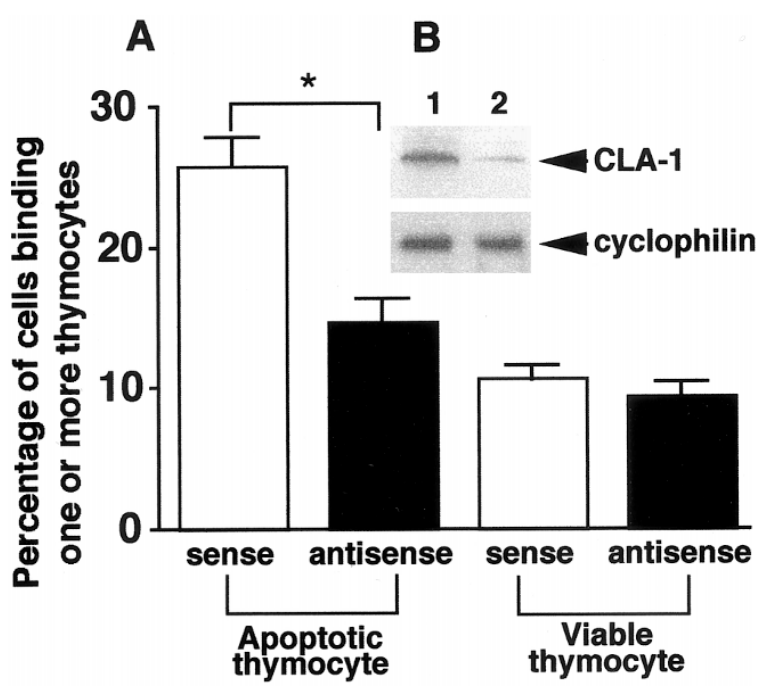

Figure 4.

Effects of hSR-B1/CLA-1 antisense oligonucleotides on binding of apoptotic thymocytes and on hSR-B1/CLA-1 protein expression in nursing TEC. A, Effects of pretreatment with sense or with antisense oligonucleotides on binding of apoptotic thymocytes. Viable or apoptotic thymocytes were added to the nursing TEC cells treated with the oligonucleotides. The binding of thymocytes by the nursing TEC was determined as described under "Methods." Results are the mean \pm SE of six experiments. *Significantly different $(p<0.005)$. B, hSR-B1/CLA-1 protein expression determined by Western blot analysis. Top: Immunoblot of hSR-B1/CLA-1 in nursing TEC. Bottom: Immunoblot of cyclophilin A as internal control. The proteins were extracted 24 hours after the last oligonucleotide treatment. Lane 1, nursing TEC treated with sense oligonucleotides; lane 2, cells treated with antisense oligonucleotides. ${ }^{*}$ Significantly different $(p<0.005)$.

SR-A, CD36, and CD68 suggest that their participation is less important. Because of the high degree of homology between CLA-1 and SR-B1, it is likely that the latter plays a similar role in other species. The role of hSR-B1/CLA-1 in phagocytosis of apoptotic cells in other tissue remains to be explored.

\section{Discussion}

Efficient removal of apoptotic cells before lysis can occur is particularly critical in the thymus, where many millions of cells die every day. This function is carried out by thymic macrophages and TNC/nursing TEC (Hiramine et al, 1996). However, the specific receptors involved and the chemical nature of the recognition signals on the apoptotic cells are only partially understood.

Apoptotic cells may display their target status in a number of different ways (Savill et al, 1993). These include changes in membrane glycoproteins leading to recognition by an uncharacterized lectin-like receptor (Duvall et al, 1985) and expression of an uncharacterized thrombospondin-binding site that allows a bridging link via thrombospondin to the vitronectin receptor $\left(a_{v} b_{3}\right.$ integrin), which is thought to act in concert with CD36 (Savill et al, 1990, 1992). A further mechanism by which phagocytes might recognize apoptotic cells was suggested by observations that macrophages bind resealed red cell ghosts and sickled red cells from patients (McEvoy et al, 1986; 
Schwartz et al, 1985). These cells have lost the normal asymmetry of membrane phospholipids, by which the outer leaflet of the membrane bilayer predominantly contains the neutral phospholipids sphingomyelin and PC, while anionic phospholipids, such as PS, are usually restricted to the inner monolayer. If such asymmetry is lost upon the induction of apoptosis, phospholipids that normally exist on the cytoplasmic side of the plasma membrane would appear on the cell surface, serving as a marker of apoptotic cells (Savill et al, 1993). Translocation of PS from the inner to the outer membrane leaflet has been reported in a variety of apoptotic cells such as thymocytes (Fadok et al, 1992b), vascular smooth muscle cells (Bennett et al, 1995), neutrophils (Liles et al, 1996), and spermatocytes (Shiratsuchi et al, 1997). The studies reported above indicate that an increase in PS on apoptotic thymocytes is involved in their recognition by nursing TEC.

Several receptors have been implicated in the recognition of apoptotic cells (Krieger, 1997; Pearson, 1996; Savill et al, 1993), including SR-A, CD36, SRB1/CLA-1, and possibly CD68. Recently Devitt et al (1998) reported that apoptotic cells interact with CD14, triggering phagocytosis of the apoptotic cells by macrophages. However, it remains unclear which of these receptors are relevant to the clearance of apoptotic thymocytes. The only one so far demonstrated to be involved was SR-A and that was in thymic macrophages, not nursing TEC. Platt et al (1996) showed that an antibody against murine SR-A inhibited phagocytosis of apoptotic thymocytes by thymic macrophages by about $50 \%$. This is consistent with the several lines of evidence suggesting that receptors that recognize OxLDL also play a role in recognizing and eliminating apoptotic cells (Krieger, 1997; Sambrano and Steinberg, 1995). For example, although SR-B1 is now identified as a receptor for high-density lipoprotein (Acton et al, 1996), it was originally placed in the scavenger receptor family on the basis of its ability to bind anionic phospholipids (Rigotti et al, 1995) and modified forms of LDL (Acton et al, 1994), including OxLDL. It also displays high homology to CD36, another receptor that binds OxLDL (Endemann et al, 1993) and participates in the recognition and uptake of apoptotic cells (Savill et al, 1992). SR-B1 has been shown to function as the PS-recognizing receptor in SR-B1-transfected cell lines such as Chinese hamster ovary cells (Fukasawa et al, 1996) and human embryonic kidney cells (Murao et al, 1997). Recent reports revealed that SR-B1 plays an important role in the phagocytosis of apoptotic rat spermatogenic cells by Sertoli cells (Shiratsuchi et al, 1999) and in the phagocytosis of apoptotic rat ovarian granulosa cells by thecal cells (Svensson et al, 1999). Together with our present results, SR-B1 or CLA-1 is the PS receptor common to the non-macrophagetype phagocytic cells (nonprofessional phagocytes).

The present studies show that hSR-B1/CLA-1, the human homolog of rodent SR-B1, is strongly expressed on human TNC/nursing TEC and that antisense hSR-B1/CLA-1 oligonucleotides significantly in- hibit the binding of apoptotic thymocytes to nursing TEC. The other receptors studied were expressed at much lower levels; CD36 was not detected at all. Antisense oligonucleotides against hSR-B1/CLA-1 suppressed hSR-B1/CLA-1 protein expression at more than $80 \%$ compared with that of sense oligonucleotide treatment, however the nursing TEC transfected with the antisense oligonucleotide showed only $40 \%$ inhibition in the binding of apoptotic thymocytes. Therefore, it might be possible that undiscovered receptors are involved in the joint binding of apoptotic thymocytes.

In summary, our results suggest that hSR-B1/CLA-1 plays a major role in the clearance of apoptotic thymocytes by nursing TEC and that this is linked at least in part to its recognition of PS on the membrane of the target cells. Because of the high degree of homology between hSR-B1/CLA-1 and rodent SR-B1, it is likely that the latter plays a similar role in other species. The role of hSR-B1/CLA-1 in phagocytosis of apoptotic cells in the thymus in vivo and in other tissues remains to be explored.

\section{Materials and Methods}

\section{Cell Culture}

Human monocyte-derived THP-1 and U973 cells (American Type Culture Collection, Rockville, Maryland) were grown in RPMI 1640 (Life Technologies, Tokyo, Japan) supplemented with $10 \%$ fetal bovine serum (FBS; Life Technologies)/antibiotics in a humidified atmosphere containing $5 \% \mathrm{CO}_{2}$.

\section{Establishment of Nursing TEC Line}

The intact thymus was obtained from a 15-year-old female patient with Hodgkin's disease during a radical surgical operation. A human nursing TEC line was established from that thymus, which was not invaded by lymphoma cells, as described (Hiramine et al, 1990, 1995). The cells were initially maintained in Eagle's MEM-D-valine medium (Life Technologies) supplemented with D-valine $(9.2 \mathrm{mg} / 100 \mathrm{ml}$; Wako, Osaka, Japan), 10\% FBS, and subsequently the nursing TEC were maintained at $37^{\circ} \mathrm{C}$ in $5 \% \mathrm{CO}_{2}$ in air at $100 \%$ humidity in DMEM medium (Life Technologies) supplemented with 10\% FBS and antibiotics.

\section{Transmission Electron Micrograph}

The nursing TEC and the coculture with the apoptotic thymocytes were fixed in $2.5 \%$ glutaraldehyde in 0.1 $\mathrm{M}$ cacodylate buffer $(\mathrm{pH} 7.4)$, postfixed in $1 \% \mathrm{OsO}_{4}$, dehydrated, and embedded in Epon 812 as described previously (Hiramine et al, 1995). Ultrathin sections were stained with $1 \%$ uranyl acetate and lead citrate and viewed with an electron microscope (JEM1200EX; JEOL, Tokyo, Japan) at $80 \mathrm{kv}$.

\section{RT-PCR}

Total RNA was isolated from nursing TEC cells as described previously (Chomczynski and Sacchi, 1987). 
Scavenger receptor mRNAs were detected by PCR analysis of the reverse transcribed RNA as described previously (Mizobuchi et al, 1994). The primer sequences for SR-A (Dufva et al, 1995), CD36 (Kashiwagi et al, 1994), and hSR-B1/CLA-1 (Calvo and Vega, 1993) were as follows: SR-A: 5' common type I and type II sense primer: 5'-TGGGAACATTCTCAGACCTTGAG-3'; 3' type I-specific antisense primer: 5'-TTGTCCATAGTGAGCTGCCTTGT-3' and 3' type II-specific primer: 5'TGCCCTAATATGATCAGTGAGTTG-3'; CD36: sense primer: 5'-CCTTCTTAGCCATITAAAGATAGC-3' and antisense primer: 5'-AGCCAGGACAGCACCAATGAC3'; hSR-B1/CLA-1: sense primer: 5'-ATGATCGTGATGGTGCCGTC-3' and antisense primer: 5'-ACTGAACCTGCAGGTGCTGA-3' . As a control, $\beta$-actin was amplified and analyzed under identical conditions using the appropriate set of primers (Fujita et al, 1994).

\section{Western Blot Analysis}

Cells were washed, scraped in PBS, and lysed as described previously (Guo et al, 1996). The proteins were resuspended under reducing conditions, and 15 $\mu \mathrm{g}$ was fractionated by size on a $7.5 \%$ SDSpolyacrylamide gel and transferred to polyvinylidene difluoride membranes for immunoblotting. The membranes were incubated for 1 hour at $4^{\circ} \mathrm{C}$ with $0.2 \%$ Tween 20 in PBS containing anti-hSR-B1/CLA-1 or SR-A antibodies (diluted 1:2000 from whole antiserum) or anti-cyclophilin A antibody (diluted 1:1000; Biomol Research, Plymouth Meeting, Pennsylvania) as described previously (Murao et al, 1997). The antibody binding was visualized by chemiluminescence detection (ECL; Amersham Pharmacia Biotech, Buckinghamshire, United Kingdom).

\section{Flow Cytometric Analysis}

U937 cells treated with 100 nM PMA (Wako) and nursing TEC were washed twice with analysis buffer (PBS containing $0.1 \% \mathrm{BSA}$ ) and resuspended to $10^{7}$ cells/ml in the same buffer. These cells were incubated with FITC-conjugated anti-CD36 antibody (1:100 dilution; Immunotech, Marseille, France) on ice for 1 hour. Cells were then washed twice with analysis buffer and resuspended in the same buffer. As a control, cells were incubated with FITC-conjugated mouse IgG (Dako Japan Co. Ltd., Kyoto, Japan). Cells were analyzed on a FACScan (Becton Dickinson, San Jose, California).

\section{Apoptotic Cell Binding and Competition Analysis}

Thymocytes were isolated from the thymus of 6-weekold BALB/c mice (Clea Japan Inc., Osaka, Japan) and washed with ice-cold RPMI 1640 supplemented with $10 \%$ FBS. To induce apoptosis, thymocytes at $5 \times 10^{6}$ cells $/ \mathrm{ml}$ were incubated with $1 \mu \mathrm{M}$ dexamethasone (Wako) in RPMI 1640/10\% FBS for 4-6 hours at $37^{\circ} \mathrm{C}, 5 \% \mathrm{CO}_{2}, 95 \%$ air, humidified. Apoptosis was confirmed by demonstrated DNA fragmentation as previously described (Hiramine et al, 1995). Nursing TEC were plated in DMEM 10\% FBS/antibiotics and allowed to adhere for 8 hours. Nursing TEC were incubated with dexamethasone-treated thymocytes in DMEM for 4 hours at $37^{\circ} \mathrm{C}$ in a humidified incubator. Nonadherent cells were removed by washing five times with DMEM, and the number of nursing TEC binding one or more thymocytes was counted as described previously (Murao et al, 1997). In competition experiments, the binding of thymocytes by the nursing TEC was determined in the presence of unilamellar vesicles consisting of phospholipids and cholesterol as described previously (Murao et al, 1997). For competition of thymocyte binding by the nursing TEC, the liposomes were added to the monolayer at a final concentration of $0.1 \mathrm{~mm}$. To inhibit the activity of CD36 and SR-A, we used mouse anti-CD36 monoclonal antibody (PharMingen, Tokyo, Japan) as a blocking antibody and SR-A inhibitor molecule, fucoidan (Sigma, St. Louis, Missouri). Nursing TEC were pretreated for 30 minutes with these inhibitors at a final concentration of $100 \mu \mathrm{g} / \mathrm{ml}$ before phagocytosing apoptotic thymocytes.

\section{Antisense Oligonucleotide Treatment}

The oligonucleotide sequences used corresponded to the $5^{\prime}$-ends of the respective mRNA. The hSR-B1/ CLA-1 sense oligomer used was 5'-CGCGCAGACATGGGCTGCTGCTCCGCC-3'; the antisense oligomer was 5'-GGCGGAGCAGCAGCCCATGTCTGCGCG-3'. Phosphorothioate oligonucleotides were purchased from Nippon Bioservice (Kanagawa, Japan). They were synthesized by a modification of the $\mathrm{H}$-phosphonate procedure and purified by ionexchange chromatography and ethanol precipitated. The cells were treated with $0.8 \mu \mathrm{m}$ oligonucleotide every 24 hours for 5 days. The proteins were extracted 24 hours after the last oligonucleotide treatment as described (Okada et al, 1997; Takeuchi et al, 1994), and the level of hSR-B1/CLA-1 expression was analyzed by Western blotting.

\section{Acknowledgements}

The authors thank K. Yuube, T. Nakagawa, and K. Yamaji for their excellent technical assistance.

\section{References}

Acton SL, Scherer PE, Lodish HF, and Krieger M (1994). Expression cloning of SR-BI, a CD36-related class B scavenger receptor. J Biol Chem 269:21003-21009.

Acton S, Rigotti A, Landschulz KT, Xu S, Hobbs HH, and Krieger M (1996). Identification of scavenger receptor SR-BI as a high density lipoprotein receptor. Science 271:518-520.

Aguilar LK, Aguilar-Cordova E, Cartwright J Jr, and Belmont JW (1994). Thymic nurse cells are sites of thymocyte apoptosis. J Immunol 152:2645-2651.

Bennett MR, Gibson DF, Schwartz SM, and Tait JF (1995). Binding and phagocytosis of apoptotic vascular smooth muscle cells is mediated in part by exposure of phosphatidylserine. Circ Res 77:1136-1142. 
Calvo D and Vega MA (1993). Identification, primary structure, and distribution of CLA-1, a novel member of the CD36/LIMPII gene family. J Biol Chem 268:18929-18935.

Chomczynski P and Sacchi N (1987). Single-step method of RNA isolation by acid guanidinium thiocyanate-phenolchloroform extraction. Anal Biochem 162:156-159.

Devitt A, Moffatt OD, Raykundalia C, Capra JD, Simmons DL, and Gregory CD (1998). Human CD14 mediates recognition and phagocytosis of apoptotic cells. Nature 392:505-509.

Dufva M, Svenningsson A, and Hansson GK (1995). Differential regulation of macrophage scavenger receptor isoforms: mRNA quantification using the polymerase chain reaction. J Lipid Res 36:2282-2290.

Duvall E, Wyllie AH, and Morris RG (1985). Macrophage recognition of cells undergoing programmed cell death (apoptosis). Immunology 56:351-358.

Endemann GL, Stanton W, Madden KS, Bryant CM, White RT, and Protter AA (1993). CD36 is a receptor for oxidized low density lipoprotein. J Biol Chem 268:11811-11816.

Fadok VA, Laszlo DJ, Noble PW, Weinstein L, Riches DW, and Henson PM (1993). Particle digestibility is required for induction of the phosphatidylserine recognition mechanism used by murine macrophages to phagocytose apoptotic cells. J Immunol 151:4274-4285.

Fadok VA, Savill JS, Haslett C, Bratton DL, Doherty DE, Campbell PA, and Henson PM (1992a). Different populations of macrophages use either the vitronectin receptor or the phosphatidylserine receptor to recognize and remove apoptotic cells. J Immunol 149:4029-4035.

Fadok VA, Voelker DR, Campbell PA, Cohen JJ, Bratton DL, and Henson PM (1992b). Exposure of phosphatidylserine on the surface of apoptotic lymphocytes triggers specific recognition and removal by macrophages. J Immunol 148:22072216.

Fadok VA, Warner ML, Bratton DL, and Henson PM (1998). CD36 is required for phagocytosis of apoptotic cells by human macrophages that use either a phosphatidylserine receptor or the vitronectin receptor (alpha $\mathrm{v}$ beta 3 ). J Immunol 161:6250-6257.

Fujita T, Yamaji Y, Sato M, Murao K, and Takahara J (1994). Gene expression of somatostatin receptor subtypes, SSTR1 and SSTR2, in human lung cancer cell lines. Life Sci 55: 1797-1806.

Fukasawa M, Adachi H, Hirota K, Tsujimoto M, Arai $\mathrm{H}$, and Inoue K (1996). SRB1, a class B scavenger receptor, recognizes both negatively charged liposomes and apoptotic cells. Exp Cell Res 222:246-250.

Greenwalt DE, Lipsky RH, Ockenhouse CF, Ikeda H, Tandon NN, and Jamieson GA (1992). Membrane glycoprotein CD36: a review of its roles in adherence, signal transduction, and transfusion medicine. Blood 80:1105-1115.

Guo Q, Wang LH, Ruan KH, and Kulmacz RJ (1996). Role of Val509 in time-dependent inhibition of human prostaglandin $\mathrm{H}$ synthase-2 cyclooxygenase activity by isoform-selective agents. J Biol Chem 271:19134-19139.

Hiramine C, Hojo K, Koseto M, Nakagawa T, and Mukasa A (1990). Establishment of a murine thymic epithelial cell line capable of inducing both thymic nurse cell formation and thymocyte apoptosis. Lab Invest 62:41-54.
Hiramine C, Nakagawa T, and Hojo K (1995). Murine nursing thymic epithelial cell lines capable of inducing thymocyte apoptosis express the self-superantigen Mls-1a. Cell Immunol 160:157-162.

Hiramine C, Nakagawa T, Miyauchi A, and Hojo K (1996). Thymic nurse cells as the site of thymocyte apoptosis and apoptotic cell clearance in the thymus of cyclophosphamidetreated mice. Lab Invest 75:185-201.

Kashiwagi H, Tomiyama Y, Kosugi S, Shiraga M, Lipsky RH, Kanayama Y, Kurat Y, and Matsuzawa Y (1994). Identification of molecular defects in a subject with type I CD36 deficiency. Blood 83:3545-3552.

Krieger M (1997). The other side of scavenger receptors: pattern recognition for host defense. Curr Opin Lipidol 8:275280.

Liles WC, Kiener PA, Ledbetter JA, Aruffo A, and Klebanoff SJ (1996). Differential expression of Fas (CD95) and Fas ligand on normal human phagocytes: implications for the regulation of apoptosis in neutrophils. J Exp Med 184:429440.

Martin SJ, Reutelingsperger CP, McGahon AJ, Rader JA, van Schie RC, LaFace DM, and Green DR (1995). Early redistribution of plasma membrane phosphatidylserine is a general feature of apoptosis regardless of the initiating stimulus: inhibition by overexpression of $\mathrm{Bcl}-2$ and Abl. J Exp Med 182:1545-1556.

McEvoy L, Williamson P, and Schlegel RA (1986). Membrane phospholipid asymmetry as a determinant of erythrocyte recognition by macrophages. Proc Natl Acad Sci USA 83: 3311-3315.

Mizobuchi M, Murao K, Takeda R, and Kakimoto Y (1994). Tissue-specific expression of isoaspartyl protein carboxyl methyltransferase gene in rat brain and testis. $J$ Neurochem 62:322-328.

Mower DA Jr, Peckham DW, Illera VA, Fishbaugh JK, Stunz LL, and Ashman RF (1994). Decreased membrane phospholipid packing and decreased cell size precede DNA cleavage in mature mouse B cell apoptosis. J Immunol 15:4832-4842.

Murao K, Terpstra V, Green SR, Kondratenko N, Steinberg D, and Quehenberger O (1997). Characterization of CLA-1, a human homologue of rodent scavenger receptor $\mathrm{BI}$, as a receptor for high density lipoprotein and apoptotic thymocytes. J Biol Chem 272:17551-17557.

Nabarra B and Papiernik M (1988). Phenotype of thymic stromal cells: An immunoelectron microscopic study with anti-IA, anti-MAC-1, and anti-MAC-2 antibodies. Lab Invest $58: 524-531$

Okada H, Danoff TM, Kalluri R, and Neilson EG (1997). Early role of Fsp1 in epithelial-mesenchymal transformation. Am J Physiol 273:F563-F574.

Pearson AM (1996). Scavenger receptors in innate immunity. Curr Opin Immunol 8:20-28.

Platt N, Suzuki H, Kurihara Y, Kodama T, and Gordon S (1996). Role for the class A macrophage scavenger receptor in the phagocytosis of apoptotic thymocytes in vitro. Proc Natl Acad Sci USA 93:12456-12460.

Raff MC (1992). Social controls on cell survival and cell death. Nature 356:397-400. 
Ramprasad MP, Fischer W, Witztum JL, Sambrano GR, Quehenberger O, and Steinberg D (1995). The 94- to 97-kDa mouse macrophage membrane protein that recognizes oxidized low density lipoprotein and phosphatidylserine-rich liposomes is identical to macrosialin, the mouse homologue of human CD68. Proc Natl Acad Sci USA 92:9580-9584.

Ren Y, Silverstein RL, Allen J, and Savill J (1995). CD36 gene transfer confers capacity for phagocytosis of cells undergoing apoptosis. J Exp Med 181:1857-1862.

Rigotti A, Acton SL, and Krieger M (1995). The class B scavenger receptors $\mathrm{SR}-\mathrm{BI}$ and $\mathrm{CD} 36$ are receptors for anionic phospholipids. J Biol Chem 270:16221-16224.

Ryeom SW, Silverstein RL, Scotto A, and Sparrow JR (1996). Binding of anionic phospholipids to retinal pigment epithelium may be mediated by the scavenger receptor CD36. J Biol Chem 271:20536-20539.

Sambrano GR and Steinberg D (1995). Recognition of oxidatively damaged and apoptotic cells by an oxidized low density lipoprotein receptor on mouse peritoneal macrophages: role of membrane phosphatidylserine. Proc Natl Acad Sci USA 92:1396-1400.

Savill J, Dransfield I, Hogg N, and Haslett C (1990). Vitronectin receptor-mediated phagocytosis of cells undergoing apoptosis. Nature 343:170-173.

Savill J, Fadok V, Henson P, and Haslett C (1993). Phagocyte recognition of cells undergoing apoptosis. Immunol Today 14:131-136.

Savill J, Hogg N, Ren Y, and Haslett C (1992). Thrombospondin cooperates with CD36 and the vitronectin receptor in macrophage recognition of neutrophils undergoing apoptosis. J Clin Invest 90:1513-1522.

Schwartz RS, Tanaka Y, Fidler IJ, Chiu DT, Lubin B, and Schroit AJ (1985). Increased adherence of sickled and phosphatidylserine-enriched human erythrocytes to cultured human peripheral blood monocytes. J Clin Invest 75:19651972.
Shiratsuchi A, Kawasaki Y, Ikemoto M, Arai H, and Nakanishi $Y$ (1999). Role of class B scavenger receptor type I in phagocytosis of apoptotic rat spermatogenic cells by Sertoli cells. J Biol Chem 274:5901-5908.

Shiratsuchi A, Umeda M, Ohba Y, and Nakanishi Y (1997). Recognition of phosphatidylserine on the surface of apoptotic spermatogenic cells and subsequent phagocytosis by Sertoli cells of the rat. J Biol Chem 272:2354-2358.

Shortman K, Vremec D, and Egerton M (1991). The kinetics of $\mathrm{T}$ cell antigen receptor expression by subgroups of $\mathrm{CD} 4^{+} 8^{+}$ thymocytes: Delineation of $\mathrm{CD}^{+} 8^{+} 3\left(2^{+}\right)$thymocytes as post-selection intermediates leading to mature T cells. J Exp Med 173:323-332.

Surh CD and Sprent J (1994). T-cell apoptosis detected in situ during positive and negative selection in the thymus. Nature 372:100-103.

Svensson PA, Johnson MS, Ling C, Carlsson LM, Billig H, and Carlsson B (1999). Scavenger receptor class B type I in the rat ovary: Possible role in high density lipoprotein cholesterol uptake and in the recognition of apoptotic granulosa cells. Endocrinology 140:2494-2500.

Takeuchi K, Sato N, Kasahara H, Funayama N, Nagafuchi A, Yonemura S, Tsukita S, and Tsukita S (1994). Perturbation of cell adhesion and microvilli formation by antisense oligonucleotides to ERM family members. J Cell Biol 125:13711384.

Wekerle H, Ketelsen UP, and Ernst M (1980). Thymic nurse cells. Lymphoepithelial cell complexes in murine thymuses: morphological and serological characterization. J Exp Med 151:925-944. 